\title{
The impact of COVID-19 pandemic on IBD endoscopic procedures in a high-volume IBD Center
}

\section{(ㄷ)(1) $\odot$}

Authors

Loris Riccardo Lopetuso ${ }^{1,2,3}$, Franco Scaldaferri ${ }^{1}$, Gianluca laniro ${ }^{1}$, Stefano Bibbò ${ }^{1}$, Carlo Romano Settanni ${ }^{1}$, Alfredo Papa $^{1}$, Alessandro Armuzzi ${ }^{1}$, Antonio Gasbarrini ${ }^{1}$, Giovanni Cammarota ${ }^{1}$

Institutions

1 UOC di Medicina Interna e Gastroenterologia; CEMAD IBD UNIT; Dipartimento di Scienze Mediche e Chirurgiche, Fondazione Policlinico Universitario “A. Gemelli” IRCCS, Roma, Italia

2 Department of Medicine and Ageing Sciences, "G. d’Annunzio" University of Chieti-Pescara, Chieti, Italia

3 Center for Advanced Studies and Technology (CAST), "G. d'Annunzio" University of Chieti-Pescara, Chieti, Italia

submitted 22.4.2020

accepted after revision 5.5 .2020

\author{
Bibliography \\ DOI https://doi.org/10.1055/a-1183-3073 | \\ Endoscopy International Open 2020; 08: E980-E984 \\ (c) Georg Thieme Verlag KG Stuttgart · New York \\ eISSN 2196-9736
}

Corresponding author

Giovanni Cammarota, UOC Medicina Interna e

Gastroenterologia, Dipartimento di Scienze Mediche e

Chirurgiche, Fondazione Policlinico Universitario A. Gemelli

IRCCS, Largo Gemelli, 8, 00168 Rome, Italy

Fax: + 0039-06-30156018

giovanni.cammarota@unicatt.it

\section{ABSTRACT}

Background and study aims The COVID-19 pandemic is having a major clinical and also organizational impact on national health care systems, particularly in high-volume hospitals that are utilized for several essential clinical needs. We aimed to analyze the short-term impact of the SARS-CoV-2 pandemic on management of endoscopic procedures in patients with inflammatory bowel disease (IBD). Patients and methods This was an observational prospective study exploring major clinical and organizational changes in endoscopic management at the IBD Center - CEMAD of the Fondazione Policlinico Gemelli IRCCS, Rome, Italy since the beginning of SARS-CoV-2 pandemic.

Results Our IBD Unit, with up to 1,500 IBD patients receiving biotechnological or experimental therapy, represents a high-volume Italian and European IBD center. Since the beginning of the outbreak, our hospital has been extremely impacted by care related to COVID-19 cases, with a consequent need to dramatically reorganize management of endoscopic procedures for IBD.

Conclusions Outbreak restrictions have significantly impacted the volume of endoscopic activities for IBD. Specific strategies have been designed to guarantee a high level of safety for both patients with IBD and healthcare personnel dedicated to their treatment.

\section{Introduction}

Coronaviruses are non-segmented, enveloped, positive-sense, single-strand RNA viruses [1] with six species known to lead to human disease. Cyclically, lethal coronaviruses have appeared in recent years. This was the case with severe acute respiratory syndrome coronavirus (SARS-CoV) in 2002 and with Middle East respiratory syndrome coronavirus in 2012. In December 2019, a new unknown species of coronavirus, then identified as SARSCoV-2, was isolated and linked to cases of pneumonia detected in Wuhan [2]. After that, more than 800,000 of cases of SARSCoV-2-related disease, called COVID-19, were diagnosed all over the world, leading the World Health Organization (WHO) to declare the infection a pandemic [3]

Italy has been one of the countries most affected by COVID19 , with more than 100,000 patients who have tested positive [3] resulting in significant and unprecedented consequences for the local health care system. Indeed, in a few weeks, the SARS-CoV-2 outbreak has become a rapid driver of striking changes in the health care system at local and national levels because it is highly contagious, often requires hospitalization and critical care assistance, and has a high rate of important mortality [4]. Infection transmission happens primarily through direct contact or air droplets and patients at higher risk are 
those with underlying chronic conditions, including diabetes, hypertension, and cardiovascular disease $[5,6]$.

In this context, inflammatory bowel diseases (IBD), such as Crohn's disease (CD) and ulcerative colitis (UC), are chronic, relapsing inflammatory disorders of the gastrointestinal tract resulting from a loss of homeostasis between the intestinal immune system and the gut microbiome in genetically-predisposed individuals [7]. Although this has not been completely described $[8,9]$, IBD patients may be more susceptible to COVID-19 because of their dysregulated immune response and immunosuppressive treatments. As the risk of severe infections (such as respiratory infections) is slightly higher in subjects receiving immunosuppressive and/or biological therapies, it is expected that the same might occur with this infection [10]. Furthermore, besides respiratory illness, the viral infection also can cause diarrhea. Indeed, SARS-CoV-2 RNA has been detected in stool specimens [11], suggesting the possibility of the capacity for infection of gastrointestinal cells and of a fecal-oral transmission route $[12,13]$. However, no specific recommendations exist for care of patients with IBD who have COVID-19 and they should treated following the same rules provided by the WHO for the general population [14].

Our IBD unit is a high-volume Italian and European IBD center and part of one of the largest university hospitals in Italy. Since the beginning of the outbreak, our country and in particular our hospital, has been extremely burdened by caring for patients with COVID-19, with a consequent need to dramatically change management of patients with IBD at every level. In the current study, we provide the first detailed observational report about the short-term impact of the SARS-CoV-2 pandemic on management of endoscopic procedures in a high-volume IBD Center.

\section{Patients and methods}

\section{Study design}

This was an observational prospective study reporting major clinical and organizational changes in endoscopic management at the IBD Center - CEMAD (Centro Malattie Apparato Digerent - Digestive Disease Center) of the Fondazione Policlinico Gemelli IRCCS, Rome, Italy from January 31, 2020 to April 5, 2020.

\section{Patients}

All patients actively followed in the IBD center were included in the study. No informed consent was required for this observational study. For each patient at the planned schedule visit/ therapeutic infusion/endoscopic procedure, the outcome of care was evaluated as either "confirmed" if no modification was observed, "delayed for clinical reasons," "delayed for patient choice," or rescheduled at a local IBD center.

\section{Physicians, nurses and other personnel involved in the study}

Working plans for all physicians, nurses, and administrative staff dedicated to endoscopic management of the IBD Unit were included in the analysis.

\section{Ethics approval}

The study protocol was conducted in accordance with the principles of the Declaration of Helsinki and approved by the Fondazione Policlinico Gemelli IRCCS Ethic Committee.

\section{Results}

\section{Impact of COVID-19 at CEMAD on IBD outpatient service}

IBD outpatient service is a good example of clinical organization for management of chronic diseases [15]. The IBD center at CEMAD Fondazione Policlinico Gemelli IRCCS is one of the largest centers in Italy by volume of patients, accounting for more than 1,500 patients actively followed for biological therapy and administration of experimental drugs. Weekly schedules include up to 150 biologic administrations, up to 20 procedures specifically for trials, and about 250 follow-up visits.

The center provides multidisciplinary evaluations with access to psychologists, psychiatrists, nutritionists, rheumatologists, dermatologists, gynecologists, abdominal and pelvic surgeons, infectious disease specialists, and other specialists. Furthermore, the center directly provides highly specialized diagnostic exams including endoscopy, surveillance endoscopy for cancer and chromoendoscopy, enteroscopy, capsule endoscopy, and intestinal ultrasound. It manages scheduling of examinations performed by other departments in the hospital, including computed tomography, magnetic resonance imaging (MRI), enterography, pelvic MRI, perianal ultrasonography, and surgical evaluation under anesthesia.

In this scenario, the COVID-19 pandemic shifted the prioritization of planning for IBD management. In our center, we considered that all visits for stable patients with IBD non-essential and could be postponed, so they were rescheduled in 8 to 12 weeks ("delayed for clinical reasons"). Conversely, all visits for IBD therapy (administration of biologic therapies and experimental therapies) or for patients who were unstable or had a relapse were judged as not eligible to be postponed. The majority of patients receiving biologic or experimental therapies were maintained on the same schedules, as were those receiving therapy as part of clinical trials ("confirmed"). However, up to $10 \%$ of followed patients decided independently to post-one their treatment ("delayed for patient choice") and up to $10 \%$ asked to be referred to a local center ("rescheduled at a local IBD center"). This resulted in an overall minimal reduction in total planned procedures.

No treatment was stopped for medical reasons. There was a reduction in clinical trial enrollment, mainly for international indications from the central study team management. Moreover, we significantly increased remote follow-up of patients ( $200 \%$ increase) by email, and by telephone. New treatments with biologics were still initiated for patients with moderate to severe IBD. Scheduled visits for unstable patients needing surgery or consultation with other specialists were also maintained. 


\section{Endoscopic setting for IBD patients during pre-COVID-19 era}

Prior to the pandemic, several standards existed for high-quality endoscopy for IBD endoscopy [16]. In our center, access to high-definition endoscopy, particularly for colon cancer surveillance, was routinely provided to patients with UC or colonic CD, as well as deep sedation during endoscopy for selected patients. Furthermore, endoscopy for IBD was mainly performed by dedicated IBD specialists experienced in use of endoscopic scores, such as the Mayo score, Rutgeerts' score for patients with CD undergoing surgery and SES-CD for patients with CD who have not undergone surgery, together with a detailed description of lesions and support of a dedicated pathologist. Finally, patients treated surgically for CD usually undergo colonoscopy to evaluate disease recurrence within 6 to 12 months. These standards are usually maintained thanks to a well-organized group that starts at the IBD outpatient clinic with a recall service for selected patients, which includes dedicated IBD nurses, an IBD endoscopic agenda, and dedicated physicians.

\section{Rearrangement of the endoscopy unit: specific implications for patients with IBD}

After the COVID-19 pandemic, our endoscopy unit, including procedures and personnel for IBD-related endoscopy, had to be reorganized quickly, for several reasons.

First, as recommended at the national and local level, only endoscopic exams forh specific indications - colorectal cancer screening, endoscopies in cancer patients, interventional endoscopies in at-risk adenomatous lesions, gastrointestinal bleedings and other emergency exams, fecal microbiota transplants for recurrent Clostridium difficile infection - and exams determined to be clinically urgent, based on physician evaluation, were performed. Accordingly, the volume of IBD-related endoscopies was decreased as well, going from 30 exams per week to approximately eight exams per week (73\% decrease, "delayed for clinical reasons"). Specifically, five main types of indications were kept ("confirmed"), including endoscopic dilations of CD strictures in symptomatic patients; push and pull enteroscopies and videocapsule endoscopies for the study of the small bowel in active and/or newly diagnosed CD; endoscopic assessment of clinically moderate-to-severe IBD reactivation; screening and follow-up endoscopies within clinical trials in active IBD patients; and fecal microbiota transplants for recurrent $C$. difficile superinfection in patients with IBD

( Fig. 1).

Each week, procedures to be prioritized in the endoscopic list were discussed among physicians in a multidisciplinary fashion, based on patient referrals to the clinical IBD center.

Once in the endoscopy waiting room area, all patients received instructions for hand hygiene, social distancing, and disclosure of fever, cough or flu0like symptoms using a COVID checklist form. Finally, all patients and individuals accompanying them (maximum one person per patient) were asked to wear surgical masks [9]. The waiting area was reorganized with all administrative procedures located close to the endoscopy

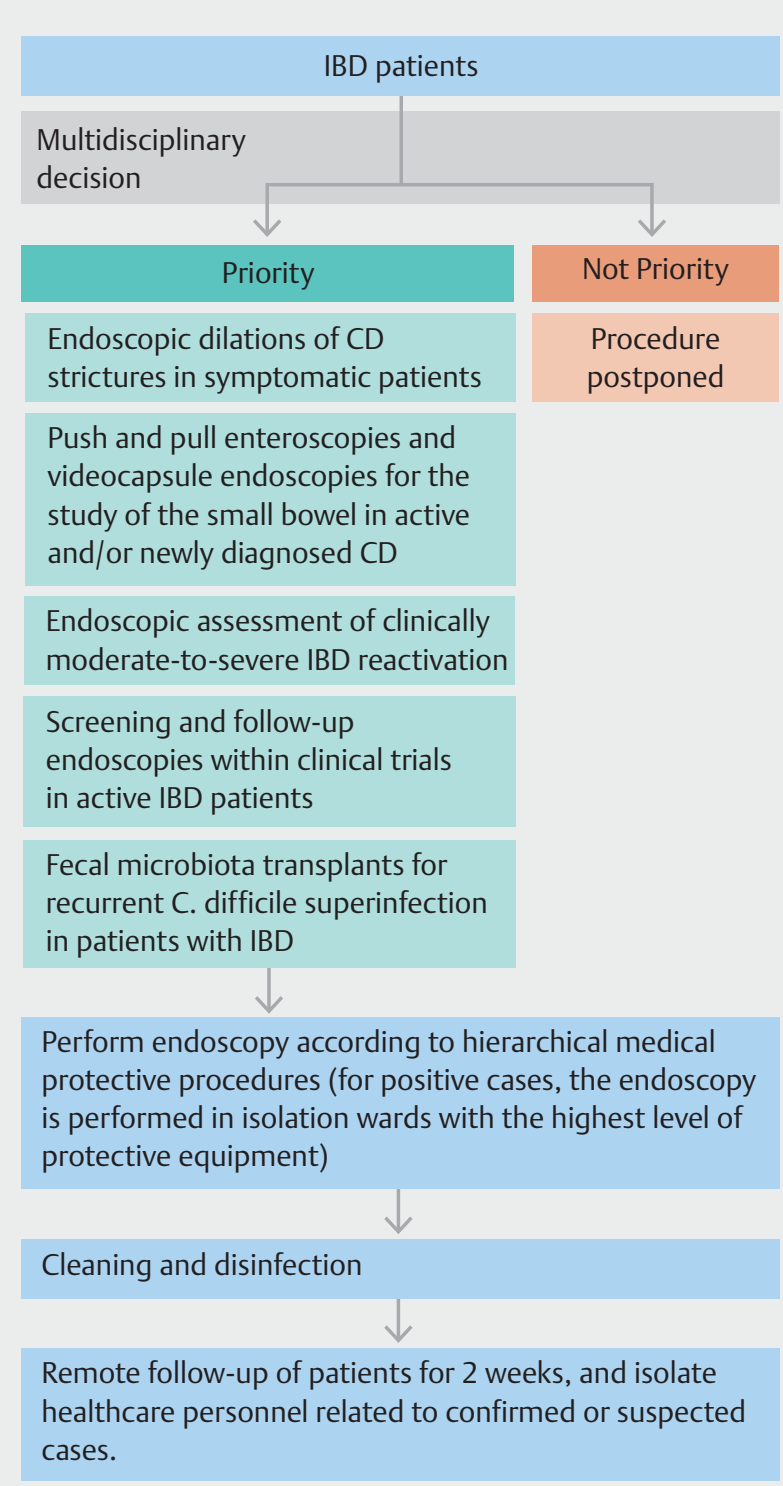

- Fig. 1 Flowchart for endoscopic procedures for IBD in a high-volume IBD unit in Italy during the COVID-19 pandemic.

waiting area in order to avoid crowd formation and to limit movements of patients through the hospital (> Fig. 2).

Another relevant change introduced was the methodology of precautions and the dress code for staff and patients. After a pre-endoscopic triage, during which nurses assessed the clinical status of a patient and presence of symptoms suggestive of COVID-19, all patients coming into the endoscopy rooms had to wear surgical masks and plastic, single-use gloves. Physicians and nurses working in the endoscopy unit had to wash their hands with soap or an alcoholic solution prior to and after interacting with patients, and wear at least an FFP1 mask and a disposable coat as personal protective equipment. There were no specific methods for dealing with patients with IBD in the endoscopy unit, so the general rules described above were followed (> Fig. 2). 


\section{Outpatients IBD}

- Remove or reduce non urgent patient accesses

- Checkpoint at entrance (surgical mask, hand wash, no companion allowed)

- COVID-19 screening (exposure and medical history, symptoms, body temperature, laboratory tests)

- If clinical suspect of COVID-19 infection immediate access to the hospital COVID-19 center

\section{Inpatients IBD}

- Postpone non urgent endoscopic procedures

- Dedicated wards and healthcare professionals trained for COVID-19

- No visitors admitted

- Standardized laboratory and radiologic exams (high definition CT scan) to exclude COVID-19

\section{COVID-19 and IBD patients}

- Postpone non urgent endoscopic procedures

- Evaluate the risk of respiratory complications compared to the benefit of the procedure

- Dedicated COVID-19 wards and dedicated healthcare professionals

- Isolation and no visitors admitted

- Dedicated radiology and invasive procedures

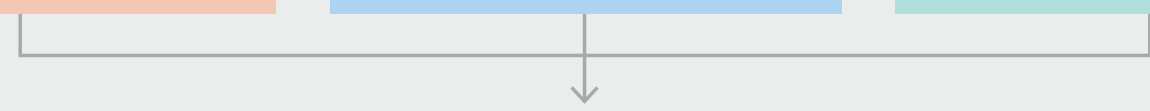

\section{ENDOSCOPY}

- Differentiate logistic pathways of patients access according to COVID-19 diagnosis

- Postpone procedure of patients with suspected or not defined COVID-19 infection

- Differentiate endoscopic and recovery rooms (individuate dedicated rooms for COVID-19 patients)

- Dedicated healthcare professionals for COVID-19

- Protective strategies for droplets in the air (wear FFP2 mask or surgical mask when appropriate, protect eyes, wear double gloves, wear shields or hats)

Fig. 2 Structural and organizational changes in a high-volume IBD unit in Italy during the COVID-19 pandemic.

Finally, the endoscopy unit had to be reorganized because of shortage staff, as several physicians were moved to COVID wards. Specifically, three endoscopists expert in IBD were temporarily redeployed to COVID wards, temporarily eliminating the clinic dedicated to endoscopy for IBD. However, that did not impact our ability to offer endoscopy to patients with IBD, the logistics of which were adjusted mainly for security reasons, to reduce risk of spreading COVID-19.

\section{Discussion}

The critical involvement of our hospital in facing this dramatic outbreak has led to massive organizational changes, with many doctors reassigned to dedicated treatment of SARS-CoV2 -positive inpatients. Social restrictions enacted by Italian national authorities beginning on March 4, 2020, consequent rules for travel limitations and social distancing, and the burden of hospitalizations and relocation of clinicians and nurses have produced resulted in drastic changes in the clinical organization of our IBD unit, and consequently, in our procedures for endoscopy in patients with IBD. This paper is the first observational detailed report about short-term impact of COVID pandemic in this field. What was observed directly regarding staff organization reflected decisions made by the risk management team, which was largely responsible for guiding the hospital during these historic conditions. On the other hand, we also tried to organize our work plans in keeping with the necessity for endoscopy. Furthermore, our work focused on patients with active IBD who were coming to the center for treatment with biological therapy and/or innovative clinical trial drugs. Overall, our patients were worried about COVID-19 and the impact of biological therapy on SARS-CoV-2 infection. Accordingly, the volume of IBD-related endoscopic procedures registered a decrease of $73 \%$, with maintenance of specific examinations that were necessary and could not be postponed.

These measures are undoubtedly guaranteeing a higher level of safety for both patients with IBD and healthcare personnel. Indeed, endoscopy should be considered a risky procedure [17]. Risk of infection of endoscopy staff and patients is linked to presence of individuals with respiratory disease spread through an airborne route. Short physical distance from patients to healthcare staff is required for endoscopic exams, but droplets from infected individuals can easily be infective with COVID-19 [17-19]. Furthermore, recent findings from SARSCoV-2 in biopsy and stool samples support potential fecal-oral transmission $[12,13]$. SARS-CoV-2 is able to enter cells through the ACE2 receptor, which is expressed more in cells from the lung and kidney, as well as in enterocytes of the ileum and co- 
Ion [6]. Considering the virus's ability to spread during the incubation period in asymptomatic individuals, methods of transmission are even more important. On the other hand, in patients for whom an endoscopic procedure could be postponed, that delay allowed them to remain at home, further reducing their likelihood of infection with COVID-19.

Current evidence does not support SARS-CoV-2 infection as a potential cause of IBD flares, but the possibility exists that they may occur. Indeed, despite the absence of gastrointestinal infection, H1N1 virus was linked to mild flares during the first week of infection, especially in UC [20]. Because the risk of SARS-CoV-2 infection is the same in the general population and as in patients with IBD, potential IBD flares may be more challenging to manage in this situation. Therefore, there was a significant need to reduce endoscopic procedures for IBD and patients were advised to continue their therapies, especially if their IBD was in remission. We believe that establishing preventive measures in an endoscopic unit dedicated to IBD is critical for guaranteeing high-quality care in a very safe setting and could help to avoid further unrecognized spread of COVID-19.

\section{Conclusion}

Optimization of endoscopy for IBD will be essential when the COVID-19 pandemic is over and an overload of postponed procedures will have to be faced. In that scenario, when the light at the end of this dramatic tunnel comes, it will be appropriate to revisit measures for infection prevention and control. The fundamental changes in physical and functional settings that we are experiencing in organization of facilities and personnel for performance of endoscopy for IBD will need to be further refined to deal not only with the prevalent risk of SARS-CoV-2 infection, but also with other new pathogens that may emerge. In this field, solid data are needed on the incidence and prognosis of COVID-19 in patients with IBD.

\section{Competing interests}

The authors declare that they have no conflict of interest.

\section{References}

[1] Weiss SR, Leibowitz JL. Coronavirus pathogenesis. Adv Virus Res 2011; 81: $85-164$

[2] World Health Organization. Pneumonia of unknown cause - China. https://www.who.int/csr/don/05-january-2020-pneumonia-of-unkown-cause-china/en/
[3] World Health Organization. Coronavirus disease (COVID-2019) situation report-72. https://www.who.int/docs/default-source/coronaviruse/situation-reports/20200401-sitrep-72-covid-19.pdf? sfvrsn=3dd8971b_2

[4] Verity R, Okell LC, Dorigatti I et al. Estimates of the severity of coronavirus disease 2019: a model-based analysis. Lancet Infect Dis 2020: doi:10.1016/S1473-3099(20)30243-7

[5] Wang C, Horby PW, Hayden FG et al. A novel coronavirus outbreak of global health concern. Lancet 2020; 395: 470-473

[6] Zhu N, Zhang D, Wang W et al. A novel coronavirus from patients with pneumonia in China, 2019. N Engl J Med 2020; 382: 727-733

[7] Bamias G, Corridoni D, Pizarro TT et al. New insights into the dichotomous role of innate cytokines in gut homeostasis and inflammation. Cytokine 2012; 59: 451-459

[8] Fiorino G, Allocca M, Furfaro F et al. Inflammatory bowel disease care in the COVID-19 pandemic era: the Humanitas, Milan experience. J Crohn Colitis 2020: doi:10.1093/ecco-jcc/jjaa058

[9] Mao R, Liang J, Shen J et al. Implications of COVID-19 for patients with pre-existing digestive diseases. Lancet Gastroenterol Hepatol 2020: doi:10.1016/S2468-1253(20)30076-5

[10] ECCO. 1st Interview COVID-19 ECCO Taskforce, published March 13, 2020.

[11] Wolfel R, Corman VM, Guggemos W et al. Virological assessment of hospitalized patients with COVID-2019. Nature 2020: doi:10.1038/ s41586-020-2196-x

[12] Xiao F, Tang M, Zheng $X$ et al. Evidence for gastrointestinal infection of SARS-CoV-2. Gastroenterology 2020: doi:10.1053/j.gastro.2020.02.055

[13] Gu J, Han B, Wang J. COVID-19: Gastrointestinal manifestations and potential fecal-oral transmission. Gastroenterology 2020: doi:10.1053/j.gastro.2020.02.054

[14] World Health Organization. Q\&A on Coronaviruses [COVID-19].

[15] Dulai PS, Singh S, Ohno-Machado L et al. Population health management for inflammatory bowel disease. Gastroenterology 2018; 154: $37-45$

[16] Fiorino G, Lytras T, Younge L et al. Quality of care standards in inflammatory bowel diseases: a European Crohn's and Colitis Organisation (ECCO) position paper. J Crohn Colitis 2020: doi:10.1093/eccojcc/jjaa023

[17] Repici A, Maselli R, Colombo M et al. Coronavirus (COVID-19) outbreak: what the department of endoscopy should know. Gastrointest Endosc 2020: doi:10.1016/j.gie.2020.03.019

[18] Tang JW, Li Y, Eames I et al. Factors involved in the aerosol transmission of infection and control of ventilation in healthcare premises. J Hosp Infect 2006; 64: 100-114

[19] Wong TW, Lee CK, Tam W et al. Cluster of SARS among medical students exposed to single patient, Hong Kong. Emerg Infect Dis 2004; 10: $269-276$

[20] Rahier JF, Papay P, Salleron J et al. Influenza A (H1N1)v infection in patients with inflammatory bowel disease: a case series. Aliment Pharmacol Therap 2011; 33: 499-500 